\title{
Caracterização fitoquímica do óleo de soja adicionado de extrato de Portulaca oleracea L.
}

\author{
Neuza Jorge ${ }^{1 *}$, Taísa Attuy Pietro ${ }^{1}$, Débora Maria Moreno Luzia ${ }^{2}$, Carolina Médici Veronezi ${ }^{1}$
}

$10.1590 / 0034-737 X 201865010001$

\begin{abstract}
RESUMO
Este trabalho teve como principais objetivos medir o teor de compostos fenólicos totais e a atividade antioxidante do extrato de Portulaca oleracea L., além de avaliar a estabilidade oxidativa e a retenção dos compostos fitoquímicos presentes no óleo de soja adicionado de antioxidantes sintéticos (TBHQ e BHT) e naturais (Extrato de Portulaca oleracea L.). Os tratamentos: óleo de soja (OS), óleo de soja adicionado de $100 \mathrm{mg} / \mathrm{kg}$ TBHQ (TBHQ), óleo de soja adicionado de $100 \mathrm{mg} / \mathrm{kg}$ BHT (BHT) e óleo de soja adicionado de $100 \mathrm{mg} / \mathrm{kg}$ extrato (Extrato) foram submetidos à termoxidação a $180^{\circ} \mathrm{C}$, por cinco horas. O extrato apresentou 99,5 mg EAG/100 g de compostos fenólicos totais e 84,6\%, 35,5\% e 1,8 $\mu \mathrm{M}$ Trolox/100 g de atividade antioxidante para DPPH, sistema $\beta$-caroteno/ácido linoleico e FRAP, respectivamente. A estabilidade oxidativa do tratamento Extrato foi inferior à do TBHQ, que apresentou o maior período de indução (7,6 h). Após aquecimento, os tratamentos TBHQ e Extrato apresentaram maior capacidade de retenção de tocoferóis e fitosteróis, atingindo valores acima de 88 e 87\%, respectivamente. Percentagens similares de ácidos graxos poli-insaturados foram encontradas nos tratamentos OS e Extrato. O extrato de beldroega na concentração $100 \mathrm{mg} / \mathrm{kg}$ foi capaz de reduzir as alterações sofridas pelo óleo de soja após aquecimento e pode ser utilizado como substituto natural de antioxidantes sintéticos.
\end{abstract}

Palavras-chave: beldroega; antioxidantes; estabilidade oxidativa; compostos bioativos.

\section{ABSTRACT}

\section{Phytochemical characterization of soybean oil with addition of Portulaca oleracea L. extract}

The main objective of the present work was to measure the total phenolic compound content and antioxidant activity of Portulaca oleracea L. extract, besides evaluating the oxidative stability and phytochemical compound retention present in soybean oil plus synthetic (TBHQ and BHT) and natural (Portulaca oleracea L. extract) antioxidants. The treatments soybean oil (SO), soybean oil plus $100 \mathrm{mg} / \mathrm{kg}$ TBHQ (TBHQ), soybean oil plus $100 \mathrm{mg} / \mathrm{kg}$ BHT (BHT), and soybean oil plus $100 \mathrm{mg} / \mathrm{kg}$ extract (Extract) were subjected to thermo-oxidation at $180^{\circ} \mathrm{C} / 5 \mathrm{~h}$. The extract presented $99.5 \mathrm{mg} \mathrm{EAG} / 100 \mathrm{~g}$ of total phenolic compounds and $84.6,35.5$, and $1.8 \mu \mathrm{M}$ Trolox $/ 100 \mathrm{~g}$ of antioxidant activity to DPPH, $\beta$-carotene/linoleic acid system, and FRAP, respectively. The oxidative stability for the Extract treatment was inferior to TBHQ, which showed the longest induction period $(7.6 \mathrm{~h})$. After heating, TBHQ and Extract treatments presented higher capacity of tocopherol and phytosterol retention, reaching values over 88 and $87 \%$, respectively. Similar percentages of polyunsaturated fatty acids were found in SO and Extract treatments. The purslane extract in the 100 $\mathrm{mg} / \mathrm{kg}$ concentration reduced the alterations suffered by soybean oil after heating and it can be used as a natural substitute for synthetic antioxidants.

Key words: purslane; antioxidants; oxidative stability; bioactive compounds.

Submetido em 23/08/2017 e aprovado em 06/02/2018

'Universidade Estadual Paulista “Júlio de Mesquita Filho", Departamento de Engenharia e Tecnologia de Alimentos, São José do Rio Preto, São Paulo, Brasil. njorge@ibilce.unesp.br ${ }^{2}$ Universidade do Estado de Minas Gerais, Departamento de Ciências Exatas e da Terra, Frutal, Minas Gerais, Brasil.

*Autor para correspondência: njorge@ibilce.unesp.br 


\section{INTRODUÇÃO}

Portulacaceae inclui cerca de 30 gêneros e 500 espécies, que se distribuem, principalmente, pelo Oeste da América do Norte, pela América do Sul e pela África, com poucos representantes na Europa e Ásia. Um dos gêneros mais representativos é a Portulaca L., com cerca de 100 espécies (Coelho \& Giulietti, 2010).

A Portulaca oleracea L., popularmente chamada de beldroega, é uma erva nutritiva, normalmente consumida na forma de salada, ou cozida em sopas. A beldroega tem sido classificada como alimento de grande poder nutricional, pois apresenta em sua composição compostos bioativos, antioxidantes, além de ser rica em ácidos graxos ômega 3, que despertam crescente interesse de pesquisadores e da indústria (Liu et al., 2000).

A beldroega pode ter efeito protetor contra o estresse oxidativo causado por deficiência de vitamina A, além de apresentar diversos efeitos biológicos benéficos, podendo ser utilizada no tratamento de doenças infecciosas, por ter atividade antifúngica e anti-inflamatória (Dkhil et al., 2011). Jin et al (2017), investigando o efeito do extrato de Portulaca oleracea sobre a formação de tumores, averiguaram que o extrato inibiu a proliferação de células cancerosas, em doses de 0,07 a 2,25 $\mu \mathrm{g} / \mathrm{mL}$. Além disso, Liu et al. (2015) sugerem que a administração de extrato etanólico de Portulaca oleracea pode ser uma estratégia eficaz para prevenir ou tratar lesões hepáticas induzidas por acetaminofeno (APAP).

O óleo de soja supre completamente as necessidades de ácidos graxos poli-insaturados do organismo, pois contém as quantidades recomendadas de ômegas 3 e 6, tão importantes para a saúde cardiovascular (Martin et al., 2006). Porém, esses mesmos ácidos graxos fazem com que o óleo de soja seja um dos mais susceptíveis aos processos oxidativos. O processo oxidativo pode provocar a perda do valor nutritivo e formar compostos prejudiciais para o organismo (Mathew \& Abraham, 2006).

Os antioxidantes são substâncias empregadas para prevenir a oxidação lipídica. Podem ser sintéticos ou naturais, e são amplamente utilizados, industrialmente. Os sintéticos apresentam alta volatilidade e instabilidade em altas temperaturas. Além disso, diversos países possuem legislação rigorosa sobre o uso de aditivos alimentares sintéticos, por causa da natureza cancerígena de alguns deles. Aliado a isso, as preferências dos consumidores mudaram e a busca por antioxidantes de fontes naturais é cada vez maior (Lobo et al., 2010).

Diante do exposto, os objetivos deste trabalho foram caracterizar o extrato de Portulaca oleracea L e avaliar o óleo de soja, adicionado do extrato, sob termoxidação a $180^{\circ} \mathrm{C}$, por cinco horas. comparando-o com os antioxidantes sintéticos TBHQ e BHT.

\section{MATERIAL E MÉTODOS}

O experimento foi conduzido no Laboratório de Óleos e Gorduras da Universidade Estadual Paulista-UNESP, em São José do Rio Preto, SP. Para sua realização, foram utilizados os antioxidantes sintéticos TBHQ (100 mg/kg) e BHT (100 mg/kg), fornecidos pela empresa Danisco S/A, de Cotia,SP, além de óleo de soja refinado, sem adição de TBHQ, e ácido cítrico, processado pela empresa Triângulo S/A, de Itápolis, SP.

A beldroega in natura (Portulaca oleracea L.), adquirida na região de São José do Rio Preto, foi selecionada, retirando-se folhas danificadas, talos e raízes. Em seguida, as folhas foram submetidas à liofilização e o material foi acondicionado em sacos de polietileno de alta densidade e mantido a $-18{ }^{\circ} \mathrm{C}$, até a realização das análises. Foram analisados os teores de umidade e lipídios, pelo método oficial proposto pela AOCS (2009).

\section{Extrato de beldroega}

Para a obtenção do extrato de beldroega, o material, liofilizado e triturado, foi extraído com álcool etílico, na proporção de 1:3 (p/v) por uma hora, sob intenso movimento em shaker, à temperatura ambiente. Em seguida, a mistura foi filtrada e armazenada, sob refrigeração, até o momento das análises. Nesse extrato, foram realizadas as análises de compostos fenólicos totais, por espectrofotometria, segundo a metodologia descrita por Singleton \& Rossi (1965); radical livre DPPH, proposto por BrandWilliams, Cuvelier \& Berset (1995); poder antioxidante de redução do ferro (FRAP), pelo método descrito por Szydlowska-Czerniak et al. (2008) e sistema $\beta$-caroteno/ ácido linoleico, de acordo com o método desenvolvido por Marco (1968) e modificado por Miller (1971).

\section{Ensaio de termoxidação}

Foram formulados cinco tratamentos, dois tratamentos com somente óleo de soja, sem (Controle) e com aquecimento (OS), e os outros com óleo de soja $+100 \mathrm{mg} / \mathrm{kg}$ de antioxidantes sintéticos (TBHQ e BHT) e naturais (Extrato), conforme descrito na Tabela 1 .

Os tratamentos foram conduzidos em chapa aquecida a $180 \pm 5^{\circ} \mathrm{C}$, utilizando-se béqueres de $50 \mathrm{~mL}$, com $30 \mathrm{~mL}$ de óleo, com relação superfície/ volume de $0,4 / \mathrm{cm}$, durante cinco horas. Após o aquecimento, as amostras foram

Tabela 1: Especificações dos tratamentos

\begin{tabular}{lc}
\hline Tratamentos & Especificações \\
\hline Controle & óleo de soja sem aquecimento \\
OS & óleo de soja com aquecimento \\
TBHQ & óleo de soja $+100 \mathrm{mg} / \mathrm{kg}$ TBHQ \\
BHT & óleo de soja $+100 \mathrm{mg} / \mathrm{kg} \mathrm{BHT}$ \\
Extrato & óleo de soja $+100 \mathrm{mg} / \mathrm{kg}$ Extrato de Beldroega \\
\hline
\end{tabular}


acondicionadas em frascos âmbar, inertizadas com nitrogênio gasoso e armazenadas, sob refrigeração, até o momento das análises.

Nos tratamentos, foram realizadas as análises de estabilidade oxidativa, segundo o método proposto pela AOCS (2009), utilizando o equipamento Rancimat (Modelo 743, Metrohm Ltd., Herisau, Switzerland), a $100{ }^{\circ} \mathrm{C}$ e com fluxo de ar de $20 \mathrm{~L} / \mathrm{h}$; composição de tocoferóis, pelo método AOCS (2009), em cromatógrafo líquido de alta eficiência (Modelo 210-263, Varian, Walnut Creek, CA, USA), com detector de fluorescência, coluna de aço inox empacotada com sílica (100 Si, Microsorb, Varian, Walnut Creek, CA, USA) e comprimentos de ondas de excitação e emissão de 290 e 330 nm, respectivamente; teores de fitosteróis, conforme AOCS (2009), em cromatógrafo gasoso (Modelo Plus-2010, Shimadzu, Chiyoda-ku, Tokyo, Japão), equipado com detector de ionização de chama, sistema de injeção split e coluna capilar de sílica fundida (Restek RTX 5, Shimadzu, Chiyoda-ku, Tokyo, Japão). As temperaturas utilizadas no injetor e no detector foram de 280 e $320^{\circ} \mathrm{C}$, respectivamente. A saponificação prévia da amostra foi conduzida de acordo com Duchateau et al. (2002).

Além disso, foi realizado o perfil de ácidos, por cromatografia em fase gasosa, dos ésteres metílicos, conforme o método da AOCS (2009), com adaptações. Foi utilizado um cromatógrafo a gás (modelo CG 3900, Varian, Walnut Creek, USA), com coluna capilar de sílica fundida (CP-Sil 88), de $60 \mathrm{~m}$ de comprimento, com diâmetro interno de 0,25 mm e espessura do filme de $0,20 \mu \mathrm{m}$ ) e as seguintes condições: gás de arraste, o hidrogênio, com velocidade linear de $30 \mathrm{~mL} / \mathrm{min}$; temperatura da coluna de 90 a $195^{\circ} \mathrm{C}$, com aumento de $10^{\circ} \mathrm{C} /$ minuto, mantida em isoterma por $16 \mathrm{mi}$ nutos; temperaturas do injetor e do detector de $230^{\circ} \mathrm{C}$ e 250 ${ }^{\circ} \mathrm{C}$, respectivamente, e razão de divisão de 1:30. A identificação dos ácidos graxos foi realizada por comparação dos tempos de retenção das amostras teste com o tempo de retenção de padrões cromatográficos de ésteres metílicos. Utilizou-se, como padrão, uma mistura composta de 37 ésteres metílicos de ácidos graxos (Supelco, Bellefonte, USA), de C4:0 a C24:1, com pureza entre 99, 1 e 99,9\%.

\section{Análise estatística}

Os resultados das determinações analíticas, em duplicata, foram submetidos à análise de variância e as diferenças entre as médias foram testadas a 5\% de probabilidade, pelo teste de Tukey, com o programa STATISTICA, versão 7.0.

\section{RESULTADOS E DISCUSSÃO}

\section{Análises no extrato}

Na Tabela 2, observa-se que o teor de umidade da beldroega in natura foi de 93,3\%, próximo aos encontra- dos por Oliveira et al. (2009) em folhas e sementes de beldroega, que apresentaram 89,2 e 92,3\%, respectivamente. Pesquisas comprovam que os teores de umidade estão relacionados com a época da colheita da planta e com as condições climáticas, como tempo seco, ameno ou póschuva (Lim \& Quah, 2007). Quanto ao teor lipídico, encontraram-se $5,1 \%$, indicando que a planta não é rica fonte de óleo.

O teor de compostos fenólicos totais obtido no extrato de beldroega foi de 99,5 mg EAG/100 g. Lim \& Quah (2007), ao analisarem compostos fenólicos totais em diferentes estádios de crescimento da Portulaca oleracea, notaram que plantas jovens apresentavam teores significativamente menores que plantas em desenvolvimento, o que pode ser explicado pela importância de compostos protetores nesse estádio, em que a atividade metabólica da planta é intensa, requerendo maior concentração de compostos essenciais para o crescimento, como antioxidantes.

A determinação da atividade antioxidante pode depender da metodologia e do radical livre gerador ou oxidante utilizado nessa medida. Por isso, é importante combinar ao menos dois ou, se possível, vários ensaios, para a obtenção de informações mais completas quanto à atividade antioxidante (Castela-Branco \& Torres, 2011). Por meio do método de sequestro do radical livre DPPH, obteve-se $84,8 \%$ de atividade antioxidante no extrato de beldroega. Oliveira et al. (2009) encontraram atividade antioxidante de $70 \%$. Os antioxidantes presentes no sistema protegem o ácido linoleico, prolongando o período de formação dos radicais livres. Para a análise FRAP, encontrou-se 1,8 $\mu \mathrm{M}$ Trolox/100 g, valor bastante pequeno, possivelmente por causa da presença de compostos pró-oxidantes interferentes. Quanto à análise de $\beta$-caroteno/ácido linoleico, foram encontrados 35,5\%, o que indica pequena diminuição na densidade óptica da amostra e, consequentemente, maior ação protetora do antioxidante, em impedir a degradação do $\beta$-caroteno.

Tabela 2: Teores de umidade e de lipídios, na beldroega in natura, e compostos fenólicos totais e capacidade antioxidante, no seu extrato

\begin{tabular}{lc}
\hline Beldroega in natura & \\
\hline Teor de umidade $(\%)$ & $93,3 \pm 0,6$ \\
Teor de lipídios $(\%)$ & $5,1 \pm 0,4$ \\
\hline Extrato & \\
\hline CFT (mg EAG/100 g) & $99,5 \pm 1,4$ \\
Atividade antioxidante & \\
$\quad$ DPPH (\%) & $84,8 \pm 0,6$ \\
$\quad$ FRAP $(\mu \mathrm{M}$ Trolox/100 g) & $1,8 \pm 0,1$ \\
$\quad \beta$-caroteno/ácido linoleico $(\%)$ & $35,5 \pm 1,1$ \\
\hline
\end{tabular}

Médias \pm desvios padrões das análises realizadas em duplicatas. 
Além disso, pode-se inferir que o extrato de beldroega tem quantidades significativas de antioxidantes lipofílicos, como tocoferóis.

\section{Análises no óleo de soja}

A estabilidade oxidativa é definida como o tempo necessário para atingir um nível de rancidez detectável, ou surpreendente mudança na taxa de oxidação (Antoniassi, 2001). Analisando-se a Tabela 3, observa-se que o TBHQ foi o tratamento mais efetivo em retardar a oxidação, resultando em maior período de indução (7,6 h). Além disso, o tratamento Extrato resultou em diferença significativa do Controle e BHT.

Segundo Luzia \& Jorge (2009), é possível monitorar a qualidade de óleos aquecidos determinando-se a concentração de antioxidantes, principalmente dos tocoferóis, que são empregados para prevenir a oxidação lipídica, consequentemente, melhorando sua estabilidade. Na Tabela 4, verifica-se que, para o isômero $\gamma$-tocol, a ação dos tratamentos TBHQ e Extrato foi mais eficiente que a do BHT, pois reteve maior quantidade desse composto. Vale ressaltar, ainda, que o Extrato resultou em

Tabela 3: Influência dos antioxidantes na estabilidade oxidativa do óleo de soja

\begin{tabular}{lc}
\hline Antioxidantes & Estabilidade oxidativa (h) \\
\hline Controle & $7,3 \pm 0,2^{\mathrm{ab}}$ \\
OS & $5,4 \pm 0,4^{\mathrm{c}}$ \\
TBHQ & $7,6 \pm 0,1^{\mathrm{a}}$ \\
BHT & $6,5 \pm 0,0^{\mathrm{ab}}$ \\
Extrato de Beldroega & $5,7 \pm 0,3^{\mathrm{bc}}$ \\
\hline
\end{tabular}

Médias \pm desvios padrões seguidas pelas mesmas letras na coluna não diferem pelo teste de Tukey ( $\mathrm{p} \geq 0,05)$. maior poder de retenção de $\delta$-tocoferol, com 74,6 mg/kg, após o aquecimento. Observa-se também, que o OS resultou em redução de 19,8\% de tocoferóis totais, em relação ao Controle. Os tratamentos TBHQ e Extrato resultaram em $88 \%$ de retenção, não havendo diferença significativa $(\mathrm{p} \geq 0,05)$ entre eles, embora ambos tenham-se diferido do BHT.

Pode-se notar, na Tabela 5, que os tratamentos. TBHQ e Extrato não diferiram estatisticamente $(p \geq 0,05)$ quanto aos teores dos compostos estigmasterol e $\beta$-sitosterol e foram mais eficientes que o BHT. É importante destacar que o Extrato resultou em maior poder de retenção de estigmasterol, com $13,5 \%$, não diferindo significativamente do Controle. Todos os tratamentos resultaram em maior quantidade do isômero $\beta$-sitosterol, seguido do campesterol e, por último, do estigmasterol, do que o OS.

O Extrato e o TBHQ foram os tratamentos que proporcionaram maiores quantidades de fitosteróis totais, seguidos de BHT e de OS. Observa-se, também, que o tratamento OS apresentou menos $22,1 \%$ do conteúdo de fitosteróis totais do que o Controle. Os tratamentos TBHQ e Extrato mostraram elevado poder de retenção com mais de $87 \%$ de fitosteróis totais.

Todos os tratamentos resultaram em dez diferentes ácidos graxos, com maior quantidade de linoleico, seguido por oleico, palmítico, $\alpha$-linolênico e esteárico (Tabela 6). Os demais ácidos graxos detectados, além de estarem em pequenas quantidades, também não apresentaram diferença significativa $(\mathrm{p} \geq 0,05)$ entre os tratamentos.

Com os tratamentos OS, BHT e Extrato foram quantificadas maiores percentagens de ácidos graxos poliinsaturados, não havendo diferença significativa $(\mathrm{p} \geq 0,05)$. Maior quantidade de ácidos graxos saturados foi detectada no tratamento TBHQ.

Tabela 4: Influência dos antioxidantes sobre os tocoferóis, em óleo de soja

\begin{tabular}{lccccc}
\hline $\begin{array}{l}\text { Tocoferóis } \\
(\mathbf{m g} / \mathbf{k g})\end{array}$ & Controle & OS & TBHQ & BHT & Extrato de Beldroega \\
\cline { 2 - 6 } & $71,7 \pm 0,1^{\mathrm{a}}$ & $57,7 \pm 0,2^{\mathrm{c}}$ & $67,3 \pm 0,1^{\mathrm{b}}$ & $67,2 \pm 0,1^{\mathrm{b}}$ & $67,5 \pm 0,2^{\mathrm{b}}$ \\
$\alpha$-tocol & $379,5 \pm 0,2^{\mathrm{a}}$ & $295,6 \pm 0,1^{\mathrm{d}}$ & $326,4 \pm 0,5^{\mathrm{b}}$ & $323,5 \pm 0,4^{\mathrm{c}}$ & $325,3 \pm 0,1^{\mathrm{b}}$ \\
$\gamma$ - tocol & $77,6 \pm 0,4^{\mathrm{a}}$ & $70,6 \pm 0,1^{\mathrm{d}}$ & $73,6 \pm 0,1^{\mathrm{c}}$ & $69,5 \pm 0,2^{\mathrm{e}}$ & $74,6 \pm 0,1^{\mathrm{b}}$ \\
$\delta$-tocol & $528,5 \pm 0,1^{\mathrm{a}}$ & $423,8 \pm 0,0^{\mathrm{d}}$ & $467,2 \pm 0,1^{\mathrm{b}}$ & $460,1 \pm 0,2^{\mathrm{c}}$ & $467,3 \pm 0,3^{\mathrm{b}}$ \\
\hline
\end{tabular}

Médias \pm desvios padrões seguidas pelas mesmas letras nas linhas não diferem pelo teste de Tukey $(\mathrm{p} \geq 0,05)$.

Tabela 5: Influência dos antioxidantes sobre os fitosteróis, em óleo de soja

\begin{tabular}{lccccc}
\hline $\begin{array}{l}\text { Fitosteróis } \\
(\mathbf{m g} / \mathbf{1 0 0} \mathbf{g})\end{array}$ & Controle & OS & TBHQ & BHT & Extrato de Beldroega \\
\cline { 2 - 6 } & $14,8 \pm 0,2^{\mathrm{a}}$ & $13,7 \pm 0,1^{\mathrm{b}}$ & $14,0 \pm 0,1^{\mathrm{b}}$ & $13,7 \pm 0,1^{\mathrm{b}}$ & $14,0 \pm 0,1^{\mathrm{b}}$ \\
\hline Campesterol & $13,8 \pm 0,1^{\mathrm{a}}$ & $12,7 \pm 0,1^{\mathrm{c}}$ & $13,2 \pm 0,2^{\mathrm{b}}$ & $13,0 \pm 0,0^{\mathrm{c}}$ & $13,5 \pm 0,1^{\mathrm{ab}}$ \\
Estigmasterol & $293,3 \pm 0,1^{\mathrm{a}}$ & $224,4 \pm 0,1^{\mathrm{d}}$ & $254,3 \pm 0,2^{\mathrm{b}}$ & $248,5 \pm 0,1^{\mathrm{c}}$ & $254,1 \pm 0,1^{\mathrm{b}}$ \\
$\beta$-sitosterol & $321,9 \pm 0,3^{\mathrm{a}}$ & $250,8 \pm 0,1^{\mathrm{d}}$ & $281,4 \pm 0,1^{\mathrm{b}}$ & $275,2 \pm 0,3^{\mathrm{c}}$ & $281,6 \pm 0,3^{\mathrm{b}}$ \\
\hline
\end{tabular}

Médias \pm desvios padrões seguidas pelas mesmas letras nas linhas não diferem pelo teste de Tukey $(\mathrm{p} \geq 0,05)$. 
Tabela 6: Influência dos antioxidantes sobre o perfil de ácidos graxos, em óleo de soja

\begin{tabular}{lcccc}
\hline $\begin{array}{l}\text { Ácidos graxos } \\
(\%)\end{array}$ & $\mathbf{O S}$ & TBHQ & Antioxidantes & Extrato de Beldroega \\
\cline { 2 - 5 } & $11,7 \pm 0,0^{\mathrm{a}}$ & $11,7 \pm 0,1^{\mathrm{a}}$ & $11,6 \pm 0,1^{\mathrm{b}}$ & $11,5 \pm 0,0^{\mathrm{c}}$ \\
\hline Palmítico & $4,1 \pm 0,0^{\mathrm{b}}$ & $4,2 \pm 0,0^{\mathrm{a}}$ & $4,1 \pm 0,0^{\mathrm{b}}$ & $4,1 \pm 0,0^{\mathrm{b}}$ \\
Esteárico & $23,6 \pm 0,1^{\mathrm{b}}$ & $23,9 \pm 0,1^{\mathrm{a}}$ & $23,5 \pm 0,1^{\mathrm{b}}$ & $23,6 \pm 0,1^{\mathrm{b}}$ \\
Oleico & $53,0 \pm 0,0^{\mathrm{a}}$ & $52,8 \pm 0,2^{\mathrm{b}}$ & $53,2 \pm 0,0^{\mathrm{a}}$ & $53,2 \pm 0,1^{\mathrm{a}}$ \\
Linoleico & $0,4 \pm 0,0^{\mathrm{a}}$ & $0,4 \pm 0,0^{\mathrm{a}}$ & $0,4 \pm 0,0^{\mathrm{a}}$ & $0,4 \pm 0,0^{\mathrm{a}}$ \\
Araquídico & $0,4 \pm 0,0^{\mathrm{a}}$ & $0,4 \pm 0,0^{\mathrm{a}}$ & $0,4 \pm 0,0^{\mathrm{a}}$ & $0,4 \pm 0,0^{\mathrm{a}}$ \\
$\gamma$-Linolênico & $0,6 \pm 0,0^{\mathrm{a}}$ & $0,6 \pm 0,0^{\mathrm{a}}$ & $0,6 \pm 0,0^{\mathrm{a}}$ & $0,6 \pm 0,0^{\mathrm{a}}$ \\
Eicosenoico & $5,5 \pm 0,0^{\mathrm{a}}$ & $5,3 \pm 0,0^{\mathrm{b}}$ & $5,5 \pm 0,0^{\mathrm{a}}$ & $5,5 \pm 0,0^{\mathrm{a}}$ \\
$\alpha$-Linolênico & $0,4 \pm 0,0^{\mathrm{a}}$ & $0,4 \pm 0,0^{\mathrm{a}}$ & $0,4 \pm 0,0^{\mathrm{a}}$ & $0,4 \pm 0,0^{\mathrm{a}}$ \\
Araquídico & $0,3 \pm 0,0^{\mathrm{a}}$ & $0,3 \pm 0,0^{\mathrm{a}}$ & $0,3 \pm 0,0^{\mathrm{a}}$ & $0,3 \pm 0,0^{\mathrm{a}}$ \\
Lignocérico & $16,8 \pm 0,0^{\mathrm{b}}$ & $17,0 \pm 0,1^{\mathrm{a}}$ & $16,8 \pm 0,6^{\mathrm{b}}$ & $16,7 \pm 0,6^{\mathrm{b}}$ \\
Saturados & $24,2 \pm 0,1^{\mathrm{a}}$ & $24,5 \pm 0,1^{\mathrm{a}}$ & $24,1 \pm 0,6^{\mathrm{a}}$ & $24,1 \pm 0,1^{\mathrm{a}}$ \\
Monoinsaturados & $59,0 \pm 0,1^{\mathrm{a}}$ & $58,5 \pm 0,2^{\mathrm{b}}$ & $59,1 \pm 0,1^{\mathrm{a}}$ & $59,1 \pm 0,1^{\mathrm{a}}$ \\
Poli-insaturados & &
\end{tabular}

Médias \pm desvios padrões seguidas pelas mesmas letras nas linhas não diferem pelo teste de Tukey $(\mathrm{p} \geq 0,05)$.

\section{CONCLUSÃO}

A aplicação do extrato de beldroega, na concentração $100 \mathrm{mg} / \mathrm{kg}$, mostrou-se mais eficiente na proteção do óleo de soja do que a do antioxidante sintético BHT. Além disso, reteve compostos bioativos, como tocoferóis e fitosteróis, igualmente ao TBHQ.

O extrato de beldroega foi capaz de reduzir as alterações sofridas pelo óleo de soja após aquecimento e pode ser indicado como substituto natural de antioxidantes sintéticos. Porém, há também a possibilidade de se utilizar o extrato de beldroega em sinergismo com outros antioxidantes, mediante novas pesquisas, a fim de serem usados na proteção de óleos vegetais sob termoxidação.

\section{REFERÊNCIAS}

Antoniassi R (2001) Métodos de avaliação da estabilidade oxidativa de óleos e gorduras. Boletim do Centro de Pesquisa e Processamento de Alimentos, 19:353-380.

AOCS - American Oil Chemists' Society (2009) Official and tentative methods of the American Oil Chemists' Society: including additions and revisions. $6^{\mathrm{a}}$ ed. Champaing, AOCS Press. 3000 p.

Brand-Williams W, Cuvelier ME \& Berset C (1995) Use of a free radical method to evaluate antioxidant activity. Food Science and Technology, 28:25-30

Castela-Branco VN \& Torres AG (2011) Capacidade antioxidante total de óleos vegetais comestíveis: determinantes químicos e sua relação com a qualidade dos óleos. Revista de Nutrição, 24:173-187.

Coelho AAOP \& Giulietti AM (2010) O gênero Portulaca L. (Portulacaceae) no Brasil. Acta Botânica Brasilica, 24:655-670.

Dkhil MA, Moniem AEA, Al-Quraishy S \& Saleh RA (2011) Antioxidant effect of purslane (Portulaca oleracea) and its mechanism of action. Journal of Medicinal Plants Research, $5: 1589-1563$.
Duchateau GSMJE, Bauer-Plank CG, Louter AJH, Van der Ham M, Boerma JA, Van Rooijen JJM \& Zanbelt PA (2002) Fast and accurate method for total 4-desmethyl sterol(s) content in spreads, fat-blends, and raw materials. Journal of the American Oil Chemists' Society, 79:273-278.

Jin H, Chen L, Wang S \& Chao D (2017) Portulaca oleracea extract can inhibit nodule formation of colon cancer stem cells by regulating gene expression of the Notch signal transduction pathway. Tumor Biology, 39:1-9.

Lim YY \& Quah EPL (2007) Antioxidant properties of different cultivars of Portulaca oleracea. Food Chemistry, 103:734-740.

Liu L, Howe P, Zhou YF, Xu ZQ, Hocart C \& Zhan R (2000) Fatty acids and b-carotene in Australian purslane (Portulaca oleracea) varieties. Journal of Chromatography A, 893:207-213.

Liu XF, Zheng CG, Shi HG, Tang GS, Wang WY, Zhou J \& Dong LW (2015) Ethanol extract from Portulaca oleracea L. attenuated acetaminophen-induced mice liver injury. American Journal of Translational Research, 7:309-318.

Lobo V, Patil A, Phatak A \& Chandra N (2010) Free radicals, antioxidants and functional foods: impact on human health. Pharmacognosy Reviews, 4:118-126.

Luzia DMM \& Jorge N (2009) Ação antioxidante do extrato de sementes de limão adicionado ao óleo de soja sob termoxidação. Revista do Instituto Adolfo Lutz, 68:27-32.

Marco G J (1968) A rapid method for evaluation of antioxidants. Journal of American Oil Chemists' Society, 45:594-598.

Martin AC, Almeida VV, Ruiz MR, Visentainer JEL, Matshushita M, Souza NM \& Visentainer JV (2006) Ácidos graxos poliinsaturados ômega-3 e ômega-6: Importância e ocorrência em alimentos. Revista de Nutrição, 19:761-770.

Mathew S \& Abraham E (2006) Studies on the antioxidant activities of cinnamon (Cinnamomum verum) bark extracts, through various in vitro models. Food Chemistry, 94:520-528.

Miller HE (1971) A simplified method for the evaluation of antioxidants. Journal of American Oil Chemists' Society, 48:9197.

Oliveira I, Valentão P, Lopes R, Andrade PB, Bento A \& Pereira JA (2009) Phytochemical characterization and radical scavenging activity of Portulaca oleracea L. leaves and stems. Microchemical Journal, 92:129-134. 
Singleton VL \& Rossi JA (1965) Colorimetry of total phenolics with phosphomolybdic-phosphotungstic acid reagents. American Journal of Enology and Viticulture, 16:144-158.

Szydlowska-Czernjak A, Karlovits G, Dianoczki C, Recseg K \& Szlyk E (2008) Comparison of two analytical methods for assessing antioxidant capacity of rapeseed and olive oils. Journal of the American Oil Chemists' Society, 85:141-149. 